\title{
BENIGN MYOCLONUS OF EARLY INFANCY
}

To redefine benign myoclonus of early infancy (BMEI), clinical and neurophysiologic features in 102 infants $(60$ male) with brief paroxysmal abnormal movements and normal neurologic and psychomotor development were studied at one center in Argentina and two in Italy. Infants with movements occurring only during sleep and those with abnormal EEG were excluded. Age at onset was 1-12 months (median 6.2 months). The nonepileptic paroxysmal motor phenomena included myoclonus in 23 , brief tonic contractions and spasms in 38, shuddering in 35, atonia or 'negative' myoclonus in 4 , and more than one motor phenomenon in 9. The movements generally involve the head and neck and upper limbs, and lower limbs are spared. EMG recordings of motor phenomena were characteristic of spasms, and associated with normal EEG. Episodes occurred only while awake in $87(85 \%)$, both awake and asleep in $15(15 \%)$, and were repeated several times a day, often ( $44 \%$ cases) in clusters. Except for 2 sisters with BMEI, no familial cases were found. Brain imaging and metabolic studies were normal. Episodes resolved at 6-30 months of age, the majority in the $2^{\text {nd }}$ year. Two cases presented clinical and EEG features of benign focal epilepsy. Language and cognitive development were normal in all cases; fifteen (16\%) developed hyperkinetic behavior without learning disorders. (Caraballo RH, Capovilla G, Vigevano F, Beccaria F, Specchio N, Fejerman N. The spectrum of benign myoclonus of early infancy: Clinical and neurophysiologic features in 102 patients. Epilepsia June 2009;50:1176-1183). (Respond: Dr Roberto H Caraballo, Neurology Department, Hospital de Pediatria, CP 1245, Buenos Aires, Argentina. E-mail: rhcaraballo@arnet.com.ar).

COMMENT. The authors conclude that the spectrum of the syndrome of BMEI is wide, each paroxysmal motor phenomenon has a characteristic EMG pattern with normal EEG, and the prognosis is benign. The syndrome is similar to that described by Lombroso CT and Fejerman N. (Ann Neurol 1977;1:138-143), and should be distinguished from West syndrome. A report of shuddering attacks in a 3-year-old girl found that flexion spasms without loss consciousness were controlled by propranolol (Barron TF, Younkin DP. Neurology 1992;42:258-259). A family history of essential tremor is reported in patients with shuddering attacks and some patients have both shuddering and tremor (Vanasse $\mathrm{M}$ et al. Neurology 1976;26:1027-1030). These authors propose that shuddering is an early manifestation of essential tremor. Intolerance to monosodium glutamate is reported in children with shuddering attacks (Reif-Leahrer L et al. N Engl J Med 1975:293:1204).

\section{POST-TRAUMATIC DANCING EPILEPSY}

Researchers at Thomas Jefferson University Hospital, Philadelphia, PA, report a case of "dancing epilepsy" in a 39-year-old, right-handed man who developed refractory complex partial seizures following head trauma at 15 years of age. During video-EEG monitoring of an episode of dancing movements with unresponsiveness lasting a few minutes, the ictal EEG was poorly localized, whereas the interictal EEG showed a left anterior temporal sharp wave focus. The MRI showed left frontal and right anterior temporal encephalomalacia and gliosis. The authors comment that dancing movements are a new behavioral manifestation of epilepsy, not typical of temporal lobe epilepsy, and more suggestive of frontal lobe epilepsy. (Bagla R, Khoury JS, Skidmore C. Teaching video neuroimages: dancing epilepsy. 